\title{
A Modified Playfair Cipher for a Large Block of Plaintext
}

\author{
V. Umakanta Sastry, N. Ravi Shankar, and S. Durga Bhavani
}

\begin{abstract}
In this paper, we have extended the analysis of the modified Playfair cipher, which includes interweaving and iteration, by considering a plaintext of any size. Here, we have carried out cryptanalysis and examined the avalanche effect. From this analysis, we have found that the cipher is a strong one and it cannot be broken by any cryptanalytic attack.
\end{abstract}

Index Terms -interweaving, inverse interweaving, substitution matrix.

\section{INTRODUCTION}

In a recent investigation [1], we have modified the Playfair cipher [2] by including interweaving and iteration. In this, the substitution table is represented in the form of a matrix of size $8 \times 16$. Further, the key consists of 64 distinct numbers, which lie between 0 and 127 . The plaintext is taken in the form of a matrix of size $8 \times 2$. Thus the size of the key is 448 bits and the size of the plaintext is 112 bits.

For a detailed account of the formation of the substitution matrix, and for the rules in the development of the cipher, one may refer to section II of [1].

In the present paper, we extend the analysis of the above cipher, by taking a plaintext of any size in general. However, we focus our attention on two cases: (1) The plaintext is a matrix of size $8 \times 8$, and (2) It is of size $8 \times \mathrm{m}$, where $\mathrm{m}$ depends upon the length of the plaintext.

Here, we notice that the substitution and the interweaving together with the iteration play a predominant role in strengthening the cipher.

In section II of this paper, we present the development of the cipher. In section III, we put forth the encryption and decryption algorithms. Then in section IV, we illustrate the cipher with a pair of examples. We discuss the cryptanalysis and Avalanche effect in sections V and VI respectively. Finally we deal with the conclusions in section VII.

\section{DEVELOPMENT OF THE CIPHER}

Consider a plaintext $\mathrm{P}$. On using the ASCII code, let it be represented in the form of a matrix of size nxm, by placing the numbers, corresponding to the plaintext characters, in a column wise manner (pad if needed).

V. Umakanta Sastry is with the Department of Computer Science and Engineering, Sreenidhi Institute of Science and Technology, India. Phone:919985012707, fax:914027640394, e-mail: vuksastry@ rediffmail.com

N. Ravi Shankar is with the Department of Computer Science and Engineering, Sreenidhi Institute of Science and Technology, Hyderabad, India.

S. Durga Bhavani is with the School of Information Technology, J.N.T. University, Hyderabad, India.
Let the plaintext matrix $\mathrm{P}$ be represented as

$\mathrm{P}=\left[\begin{array}{cccc}P_{11} & P_{12} & \ldots & P_{1 m} \\ P_{21} & P_{22} & \ldots & P_{2 m} \\ \cdot & \cdot & \cdots & \cdot \\ \cdot & \cdot & \cdots & \cdot \\ \cdot & \cdot & \cdots & \cdot \\ P_{n 1} & P_{n 2} & \cdots & P_{n m}\end{array}\right](1)$

Let us now describe the process of substitution. To this end, we focus our attention on the first two columns of this matrix. On using the set of substitution rules (mentioned in [1]), the matrix $\mathrm{P}$ assumes the form

\begin{tabular}{|c|c|c|c|c|}
\hline$Q_{11}$ & $Q_{12}$ & $P_{13}$ &.. & $P_{1 m}$ \\
\hline$Q_{21}$ & $Q_{22}$ & $P_{23}$ & $\cdots$ & $P_{2 m}$ \\
\hline$\cdot$ & . & $\cdot$ & $\cdots$ & · \\
\hline - & . & . & $\cdots$ & $\cdot$ \\
\hline . & . & . & $\ldots$ & . \\
\hline$Q_{n 1}$ & $Q_{n 2}$ & $P_{n 3}$ & $\cdots$ & $P_{n m}$ \\
\hline
\end{tabular}

where Qs are the elements obtained on substitution.

We now take the third and fourth columns of the $\mathrm{P}$, and carryout the substitution process by using the substitution matrix. In a similar manner, we perform the substitution to the pairs of columns $(5,6),(7,8)$ and so on till we exhaust all the columns. However, if the plaintext matrix contains odd number of columns, we pad it by including eight more additional characters, so that the number of columns becomes even. Then the matrix assumes its final form at the end of the substitution, denoted by Q.

We now apply the process of interweaving on the matrix obtained above. Firstly, we convert the elements of Q into their binary form. Since each element of $Q$ lies between 0 and 127 , it can be represented in terms of seven binary bits. Thus we have

$\mathrm{b}=\left[\begin{array}{cccccc}b_{11} & b_{12} & \cdots & b_{17} & m \\ b_{21} & b_{22} & \cdots & b_{27} & m \\ \cdot & \cdot & \cdots & \cdot \\ \cdot & \cdot & \ldots & \cdot \\ \cdot & \cdot & \cdots & \cdot \\ b_{n 1} & b_{n 2} & \cdots & b_{n}{ }_{n} m\end{array}\right]$

We now take the first column of $b$, and give a circular rotation, so that it assumes the form $\left[b_{21}, b_{31}, b_{41}, \ldots b_{n 1}, b_{11}\right]^{T}$, 
where $\mathrm{T}$ denotes the transpose of the vector.

Here, each element of the column is moved up by one row with the element in the first row circularly following the last. We apply similar procedure on all the odd numbered columns. We then apply a circular left shift by one position on all the even numbered rows.

Converting the matrix $\mathrm{b}$ into its decimal form, we get the modified Q.

On applying the aforementioned processes, i.e., substitution and interweaving for $\mathrm{N}$ rounds, we get the ciphertext $\mathrm{C}$. This completes the process of encryption. The process of decryption is opposite to that of encryption. The reverse process of interweaving is called as inverse interweaving and that of substitution as reverse substitution. These are employed in the process of decryption. The schematic diagram describing the cipher is given in Fig. 1

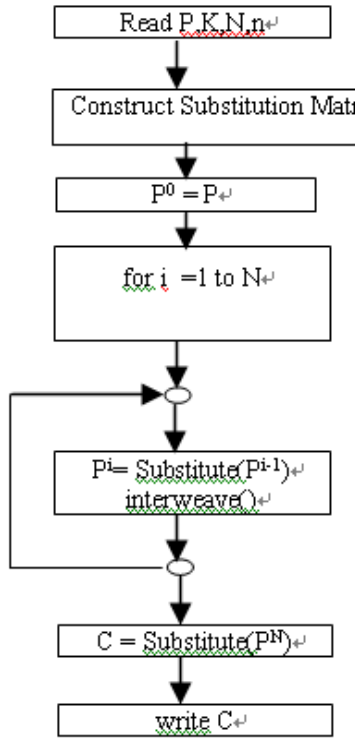

a) Encryption

Fig. 1. Schematic

In this analysis, $\mathrm{N}$ denotes the number of iterations and it is taken as 16 Algorithms

\section{A. Algorithm for encryption}

1. $\operatorname{read~n,N,K,P;}$

2. Construct Substitution matrix

3. $\mathrm{P}^{0}=\mathrm{P}$;

4. for $\mathrm{i}=1$ to $\mathrm{N}\{$

$\mathrm{P}^{\mathrm{i}}=\operatorname{Substitute}\left(\mathrm{P}^{\mathrm{i}-1}\right)$

interweave();

\}

5. $\mathrm{C}=$ Substitute $\left(\mathrm{P}^{\mathrm{N}}\right)$;

6. write C;

B. Algorithm for decryption

1. read n,N,K,C;

2. Construct Substitution matrix

3. $\mathrm{P}^{\mathrm{N}}=$ reverse substitute $(\mathrm{C})$;

4. for $\mathrm{i}=\mathrm{N}$ to 1\{

invinterweave();

$\mathrm{P}^{\mathrm{i}-1}=$ reverse substitute $\left(\mathrm{P}^{\mathrm{i}}\right) ;$ \}

5. $\mathrm{P}=\mathrm{P}^{0}$;
6. write P;

C. Algorithm for interweave

1. construct $\left[b_{i j}\right], i=1$ ton, $j=1$ to $7 m$ from $P$;

2. for $\mathrm{j}=1$ to $7 \mathrm{~m}$ in step 2 \{

$$
\mathrm{k}=\mathrm{b}_{1 \mathrm{j}} \text {; }
$$

for $\mathrm{i}=1$ to $\mathrm{n}-1$ \{

$$
\mathrm{b}_{\mathrm{ij}}=\mathrm{b}_{(\mathrm{i}+1) \mathrm{j}} \text {; }
$$

\}

$\mathrm{b}_{\mathrm{nj}}=\mathrm{k}$;

\}

3. for $i=2$ to $n$ in step 2\{

$\mathrm{k}=\mathrm{b}_{\mathrm{i} 1}$;

for $\mathrm{j}=1$ to $7 \mathrm{~m}-1$ \{

$$
\mathrm{b}_{\mathrm{ij}}=\mathrm{b}_{\mathrm{i}(\mathrm{j}+1)} \text {; }
$$

\}

$\mathrm{b}_{\mathrm{in}}=\mathrm{k}$;

\}

4. Construct $\mathrm{P}$ from $\mathrm{b}_{\mathrm{ij}}$;

D Algorithm for invinterweave

1. construct $\left[b_{\mathrm{ij}}\right], \mathrm{i}=1$ to $8, \mathrm{j}=1$ to $7 \mathrm{~m}$ from $\mathrm{P}$;

2. for $i=n$ to 2 in step 2 \{

$\mathrm{k}=\mathrm{b}_{\mathrm{i} 7 \mathrm{~m}}$;

for $\mathrm{j}=7 \mathrm{~m}$ to 2\{

$\mathrm{b}_{\mathrm{ij}}=\mathrm{b}_{\mathrm{i}(\mathrm{j}-1)}$;

\}

$\mathrm{b}_{\mathrm{il}}=\mathrm{k}$;

\}

3. for $\mathrm{j}=7 \mathrm{~m}-1$ to 1 in step 2\{

$\mathrm{k}=\mathrm{b}_{\mathrm{nj}}$;

for $\mathrm{i}=\mathrm{n}$ to 2 \{

$\mathrm{b}_{\mathrm{ij}}=\mathrm{b}_{(\mathrm{i}-1) \mathrm{j}}$;

\}

$\mathrm{b}_{1 \mathrm{j}}=\mathrm{k}$;

\}

4. Construct $\mathrm{P}$ from $b_{\mathrm{ij}}$;

\section{ILlUSTRATION OF THE CIPHER}

Consider the plaintext given below.

No one shall forget the past. The destruction of Hiroshima and Nagasaki, as the destiny speaks, shall be remembered for ever. Whenever we think of the development of the nuclear energy, we must fully feel that it should be utilized for the welfare of the mankind. Transmit this message as safely as you can.

Let us focus our attention on the first sixty four characters of the plaintext given by

No one shall forget the past. The destruction of Hiroshima andp

This plaintext, in its ASCII representation, when arranged in the form of an $8 \times 8$ matrix, assumes the form

$\mathrm{P}=\left[\begin{array}{llllllll}78 & 104 & 103 & 112 & 104 & 117 & 102 & 105 \\ 111 & 97 & 101 & 97 & 101 & 99 & 32 & 109 \\ 32 & 108 & 116 & 115 & 32 & 116 & 72 & 97 \\ 111 & 108 & 32 & 116 & 100 & 105 & 105 & 32 \\ 110 & 32 & 116 & 46 & 101 & 111 & 114 & 97 \\ 101 & 102 & 104 & 32 & 115 & 110 & 111 & 110 \\ 32 & 111 & 101 & 32 & 116 & 32 & 115 & 100 \\ 115 & 114 & 32 & 84 & 114 & 111 & 104 & 32\end{array}\right]$

(5)

On adopting the procedure described in section II of [1], 
we get the substitution matrix given by (6).

$\left[\begin{array}{llllllllllllllll}53 & 62 & 124 & 33 & 49 & 118 & 117 & 43 & 45 & 12 & 63 & 29 & 60 & 35 & 58 & 11 \\ 8 & 41 & 46 & 30 & 108 & 102 & 115 & 51 & 47 & 119 & 38 & 42 & 112 & 99 & 27 & 61 \\ 57 & 120 & 6 & 31 & 116 & 26 & 122 & 125 & 56 & 37 & 113 & 52 & 3 & 54 & 15 & 121 \\ 36 & 40 & 44 & 10 & 19 & 109 & 105 & 4 & 114 & 111 & 83 & 50 & 74 & 0 & 107 & 28 \\ 1 & 2 & 5 & 7 & 9 & 13 & 14 & 16 & 17 & 18 & 20 & 21 & 22 & 23 & 24 & 25 \\ 32 & 34 & 39 & 48 & 55 & 59 & 64 & 65 & 66 & 67 & 68 & 69 & 70 & 71 & 72 & 73 \\ 75 & 76 & 77 & 78 & 79 & 80 & 81 & 82 & 84 & 85 & 86 & 87 & 88 & 89 & 90 & 91 \\ 92 & 93 & 94 & 95 & 96 & 97 & 98 & 100 & 101 & 103 & 104 & 106 & 110 & 123 & 126 & 127\end{array}\right]$

On employing the inverse interweaving process, we get the

On applying the substitution process, we get the modified modified $\mathrm{P}^{\mathrm{N}}$ as

plaintext, denoted by $\mathrm{P}^{1}$, as

$\mathrm{P} 1=\left[\begin{array}{llllllll}86 & 95 & 110 & 119 & 98 & 63 & 115 & 109 \\ 105 & 103 & 103 & 98 & 123 & 47 & 59 & 36 \\ 55 & 8 & 122 & 108 & 55 & 57 & 59 & 126 \\ 19 & 119 & 55 & 57 & 98 & 4 & 36 & 64 \\ 92 & 70 & 6 & 108 & 103 & 114 & 109 & 101 \\ 97 & 47 & 92 & 68 & 112 & 98 & 74 & 103 \\ 67 & 36 & 92 & 66 & 57 & 55 & 51 & 98 \\ 47 & 105 & 66 & 75 & 111 & 83 & 92 & 68\end{array}\right]$

(7)

On using the process of interweaving, we get the transformed plaintext as

$$
\mathrm{P} 1=\left[\begin{array}{llllllll}
121 & 31 & 77 & 69 & 84 & 95 & 118 & 89 \\
99 & 34 & 111 & 102 & 115 & 59 & 59 & 116 \\
47 & 59 & 100 & 114 & 110 & 88 & 99 & 84 \\
25 & 102 & 23 & 108 & 98 & 80 & 44 & 69 \\
104 & 14 & 25 & 89 & 75 & 69 & 31 & 75 \\
67 & 46 & 92 & 64 & 120 & 55 & 98 & 98 \\
87 & 67 & 45 & 6 & 119 & 102 & 51 & 77 \\
7 & 125 & 106 & 95 & 103 & 23 & 118 & 69
\end{array}\right]
$$

(8)

After carrying out all the 16 iterations, we get the ciphertext $\mathrm{C}$ in the form

$$
\mathrm{C}=\left[\begin{array}{llllllll}
42 & 56 & 40 & 14 & 126 & 122 & 36 & 65 \\
86 & 114 & 87 & 116 & 123 & 44 & 42 & 60 \\
31 & 114 & 55 & 9 & 105 & 5 & 89 & 16 \\
28 & 119 & 40 & 47 & 123 & 36 & 52 & 46 \\
1 & 110 & 120 & 55 & 2 & 90 & 68 & 81 \\
71 & 59 & 79 & 44 & 67 & 69 & 99 & 89 \\
81 & 108 & 88 & 99 & 116 & 20 & 82 & 115 \\
64 & 31 & 11 & 17 & 18 & 22 & 35 & 94
\end{array}\right]
$$

In the process of decryption, we take the cipher text $\mathrm{C}$, obtained above, and apply the reverse substitution procedure. Thus we get

$$
\mathrm{PN}=\left[\begin{array}{llllllll}
47 & 52 & 105 & 2 & 98 & 15 & 4 & 32 \\
84 & 83 & 79 & 52 & 94 & 0 & 112 & 29 \\
56 & 10 & 9 & 19 & 44 & 14 & 82 & 23 \\
111 & 61 & 114 & 41 & 92 & 0 & 6 & 42 \\
22 & 92 & 116 & 34 & 24 & 76 & 64 & 86 \\
70 & 55 & 77 & 19 & 66 & 68 & 35 & 71 \\
79 & 115 & 89 & 112 & 113 & 9 & 81 & 51 \\
48 & 122 & 45 & 25 & 17 & 21 & 124 & 123
\end{array}\right]
$$

$$
\mathrm{PN}=\left[\begin{array}{llllllll}
53 & 90 & 60 & 17 & 17 & 23 & 104 & 81 \\
86 & 82 & 101 & 97 & 116 & 5 & 82 & 24 \\
84 & 81 & 14 & 28 & 94 & 2 & 33 & 31 \\
77 & 45 & 80 & 105 & 86 & 5 & 44 & 43 \\
43 & 63 & 50 & 1 & 12 & 34 & 2 & 42 \\
78 & 38 & 111 & 19 & 72 & 4 & 33 & 3 \\
71 & 61 & 76 & 57 & 18 & 68 & 98 & 77 \\
50 & 123 & 45 & 88 & 57 & 68 & 124 & 123
\end{array}\right]
$$

After carrying out all the sixteen iterations, we get the plaintext in the form

$$
\mathrm{P}=\left[\begin{array}{llllllll}
78 & 104 & 103 & 112 & 104 & 117 & 102 & 105 \\
111 & 97 & 101 & 97 & 101 & 99 & 32 & 109 \\
32 & 108 & 116 & 115 & 32 & 116 & 72 & 97 \\
111 & 108 & 32 & 116 & 100 & 105 & 105 & 32 \\
110 & 32 & 116 & 46 & 101 & 111 & 114 & 97 \\
101 & 102 & 104 & 32 & 115 & 110 & 111 & 110 \\
32 & 111 & 101 & 32 & 116 & 32 & 115 & 100 \\
115 & 114 & 32 & 84 & 114 & 111 & 104 & 32
\end{array}\right]
$$

The ciphertext corresponding to the entire plaintext (taken as blocks of 64 characters), given in (3), in hexadecimal notation, is

14A0D71B4E82199113E8D2AFB01C541E081B70390B42 20CB1AD6E0A12FDF882AADF1A1498C997D0EB4767F E1CF206DAE946ED6CF49F00CDC5211BF1AD19088986 2349123053D1E98A29DE314D7ACA4FB2C177637CB41 926D02A45512A534C9C4C1159F9F33A00FE187FA6D4 E77A22573C13948B7EF9CB3B4952CD1831587B165F95 0DE536EC81492C712A15E.

(13)

We now take another example, wherein the entire plaintext, given in (3), is taken as a single block, consisting of 312 characters. Let us now pad the plaintext by including eight more characters, say, $s, t, u, v, w, x, y, z$. Then the plaintext, consisting of 320 characters, is arranged in the form of a matrix of size $8 \times 40$. For convenience, this is represented as $\mathrm{P}=[\mathrm{AB}]$, where $\mathrm{A}$ and $\mathrm{B}$ are given in (14) and (15) respectively.

The ciphertext corresponding to the above mentioned plaintext (taken as a single block of 320 characters), in its hexadecimal notation, is obtained as

E7C24E39557A51FD112BB453F2CCA78E0EE3F6E80 278D85DE7F668613DEBCA24925B91CEF6D9413026CA 
1D10D221B0CD0F8B5FE77D456124F7E9C0172333A9D FD49CDBF8056A1B748EA2418FC0AEFFEB900ACD215 B7725801F6A3C5AAEE3277FC89400C16BAAF7313B42

\begin{tabular}{|c|c|c|c|c|c|c|c|c|c|}
\hline 8 & 104 & 103 & 112 & 104 & 117 & 102 & 105 & 78 & 44 \\
\hline 111 & 97 & 101 & 97 & 101 & 99 & 32 & 109 & 97 & 32 \\
\hline 32 & 108 & 116 & 115 & 32 & 116 & 72 & 97 & 103 & 97 \\
\hline 111 & 108 & 32 & 116 & 100 & 105 & 105 & 32 & 97 & 115 \\
\hline 110 & 32 & 116 & 46 & 101 & 111 & 114 & 97 & 115 & 32 \\
\hline 101 & 102 & 104 & 32 & 115 & 110 & 111 & 110 & 97 & 116 \\
\hline 32 & 111 & 101 & 32 & 116 & 32 & 115 & 100 & 107 & 104 \\
\hline 115 & 114 & 32 & 84 & 114 & 111 & 104 & 32 & 105 & 101 \\
\hline 12 & 32 & 108 & 114 & 109 & 108 & 116 & 115 & 101 & 101 \\
\hline 109 & 116 & 101 & 103 & 117 & 121 & 104 & 104 & 32 & 100 \\
\hline 101 & 104 & 97 & 121 & 115 & 32 & 97 & 111 & 117 & 32 \\
\hline 110 & 101 & 114 & 44 & 116 & 102 & 116 & 117 & 116 & 102 \\
\hline 116 & 32 & 32 & 32 & 32 & 101 & 32 & 108 & 105 & 111 \\
\hline 32 & 110 & 101 & 119 & 102 & 101 & 105 & 100 & 108 & 114 \\
\hline 111 & 117 & 110 & 101 & 117 & 108 & 116 & 32 & 105 & 32 \\
\hline 102 & 99 & 101 & 32 & 108 & 32 & 32 & 98 & 122 & 116 \\
\hline
\end{tabular}

that the ciphertext obtained above can be brought back to its original form by applying the decryption process.

\section{CRYPTANALYSIS}

It is well known to us that the general types of cryptanalytic attacks are (1) Ciphertext only (Brute force) attack, (2) Known plaintext attack and (3) Chosen plaintext/ciphertext attack. When the ciphertext is known to us, we take various plaintexts one after another and try to see if any one of the plaintexts taken by us yields the ciphertext under consideration. In this problem, when the size of the plaintext matrix is $8 \times 8$ i.e. 448 binary bits, the different possible plaintexts which we have to make use of, are $2^{448}$ $\left(\approx 10^{134.4}\right)$. As this is a very large number, the cipher cannot be broken by the brute force attack. When the size of the plaintext matrix is immensely large (i.e., in the case of the plaintext matrix of size $8 \times 40$ ), this brute force attack is totally ruled out.

In this problem, the key consists of 64 distinct numbers, where each number lies between 0 and 127. Thus the size of the key space is ${ }^{128} P_{64}$. Hence it is impossible to find the plaintext corresponding to the given ciphertext by exhausting the computation with all possible keys.

Now let us consider the known plaintext attack. In this case, we know as many plaintext and ciphertext pairs as we require. As we know the plaintext at the beginning of the first iteration, and the ciphertext at the end of the last iteration, linking them directly in any manner and determining the key in any way is totally impossible, as there are a number of transformations in between.

A choice of the plaintext or a choice of the ciphertext for the determination of the key cannot be done as the plaintext undergoes a number of transformations at various stages of the iterative process.

Thus this approach also is not of any use.

In the light of the above facts, we conclude that this cipher
A698A7B0809D5F95FCCDC89FA82BCE9E94DFBCC26 B6CC1CC713DD61353FDF6AE4483. It can be verified

$\left.\begin{array}{llllllllll}32 & 32 & 32 & 101 & 101 & 32 & 32 & 114 & 110 & 101 \\ 100 & 115 & 115 & 32 & 114 & 101 & 87 & 32 & 107 & 32 \\ 101 & 112 & 104 & 114 & 101 & 118 & 104 & 119 & 32 & 100 \\ 115 & 101 & 97 & 101 & 100 & 101 & 101 & 101 & 111 & 101 \\ 116 & 97 & 108 & 109 & 32 & 114 & 110 & 32 & 102 & 118 \\ 105 & 107 & 108 & 101 & 102 & 46 & 101 & 116 & 32 & 101 \\ 110 & 115 & 32 & 109 & 111 & 32 & 118 & 104 & 116 & 108 \\ 121 & 44 & 98 & 98 & 114 & 32 & 101 & 105 & 104 & 111\end{array}\right]$ (14)

is a strong one and it cannot be broken by any cryptanalytic attack.

\section{AVALANCHE EFFECT}

On using the ASCII code, the plaintext, given in (4), can be represented in its binary form as 100111011010001100111111000011010001110101110011 011010011101111110000111001011100001110010111000 110100000110110101000001101100111010011100110100 000111010010010001100001110111111011000100000111 010011001001101001110100101000001101110010000011 101000101110110010111011111110010110000111001011 100110110100001000001110011110111011011111101110 010000011011111100101010000011101000100000111001 111001001110011111001001000001010100111001011011 1111010000100000 .

(16)

If we change the $4^{\text {th }}$ character from $\mathbf{o}$ to $\mathbf{n}$ (i.e., from ASCII code 111 to 110), the plaintext will be in the form 100111011010001100111111000011010001110101110011 011010011101111110000111001011100001110010111000 110100000110110101000001101100111010011100110100 000111010010010001100001110111011011000100000111 010011001001101001110100101000001101110010000011 101000101110110010111011111110010110000111001011 100110110100001000001110011110111011011111101110 010000011011111100101010000011101000100000111001 111001001110011111001001000001010100111001011011 1111010000100000 .

(17)

It can be seen that the plaintexts, given in (16) and (17), differ by one bit.

The ciphertexts corresponding to the above plaintexts are 000101001010000011010111000110110100111010000010 000110011001000100010011111010001101001010101111 101100000001110001010100000111100000100000011011 011100000011100100001011010000100010000011001011 000110101101011011100000101000010010111111011111 100010000010101010101101111100011010000101001001 
100011001001100101111101000011101011010001110110 011111111110000111001111001000000110110110101110 100101000110111011010110110011110100100111110000 0000110011011100

(18)

and

100111001111111100111000000011000000001011001100 001000010101001100110101000011011010101011110001 001010100011111111001111100010111110000010001000 001111010101001101010111011101010001001100101111 101010111001101000011110010001110101100000110010 100001000101010111010010110011100101000011010010 111000111011010100011001101100100110010000101110 010100000001100011001101101010000111111011100000 000111111101001011100010111000100001100100101110 0010111010110000 .

(19)

It can be readily verified that the ciphertexts, given in (18) and (19), differ by 235 bits. This is quite considerable.

We now change the key element $\mathrm{K}_{22}$ (i.e., second row and sixth column of (6)) from 102 to 103 . With this change, the key changes by one bit. On applying the modified key on the original plaintext, given in (5), we get the corresponding ciphertext as

111100111100111011111100111000010100011000111010 100101101000000101111000111010110001001100110101

000000011001111101001011111001010011011110001100 001101001011001000101110100000011111101101110000 110100010001010010111111001011000000101001000011 011010001101010010011000000100010101111001110100 010011110001000101101100110100111010111101000000 100000010111111110100000111000010000000111111111 111100111011001101010010011110000110111000010101 1100000000001100 .

(20)

The ciphertexts given in (18) and (20) differ by 241 bits which is conspicuous.

From the above analysis, we notice that this cipher produces strong avalanche effect.

\section{CONCLUSIONS}

In this paper, we have extended the analysis of modified Playfair cipher by taking a very large plaintext into consideration. Here, pairs of characters are taken from the adjacent columns (characters are taken from $1^{\text {st }}$ and $2^{\text {nd }}$ columns, $3^{\text {rd }}$ and $4^{\text {th }}$ columns, etc.) of the plaintext matrix for the purpose of substitution. The process of interweaving and the process of substitution modify the plaintext at each stage of the iteration. This causes confusion and diffusion in a systematic manner and enhances the strength of the cipher.

The algorithms developed in this analysis for encryption and decryptions, along with the other requisites, are implemented in $\mathrm{C}$ language.

The time required for the encryption of the entire plaintext in (3), (taken as a single block) is $20.5^{*} 10^{-3}$ seconds and that of the decryption is $20.5^{*} 10^{-3}$ seconds.

From the cryptanalysis, and the avalanche effect carried out in this analysis, we conclude that the cipher is a potential one, and it cannot be broken by any cryptanalytic attack.

\section{REFERENCES}

[1] 1. VUK Sastry, N.Ravi Shankar, "A Modified Playfair Cipher Involving Interweaving and Iteration", (accepted for publication) International Journal of Computer Theory and Engineering, to be published.

[2] 2. William Stallings, "Cryptography and Network Security: Principles and Practices", Third edition, Chapter 2, pp.35.

[3] Biographical notes:

[4] V. Umakanta Sastry was formerly a professor at Indian Institute of Technology, Kharagpur, India. Presently he is the Director, School of Computer Science and informatics and Dean (R\&D) at Sree Nidhi Institute of Science and Technology, Hyderabad, India. He is currently guiding a number of Research Scholars for Ph.d in the areas of Information Security and Image Processing.

[5] N. Ravi Shankar is a Professor and is currently heading the department of Computer Science and Engineering, Sree Nidhi Institute of Science and Technology, Hyderabad, India. He is actively engaged in research in the area of Information Security.

[6] S. Durga Bhavani obtained her Ph.D from University of Hyderabad, India in the area of Evidential Reasoning. She is presently a Professor in Computer Science \& Engineering, School of Information Technology, JNT University, Hyderabad, India. Her research interests are applications of uncertain reasoning techniques and Information Security. 